\title{
CALIDAD DE PRODUCTOS FARMACÉUTICOS Y AFINES EN EL PERÚ PESQUISADOS POR DIGEMID, 2002 - 2006
}

\author{
Quality of pharmaceutical and related products in Peru inspected by DIGEMID, 2002-2006
}

\author{
María Margarita Coral Monge ${ }^{1}$, José Juárez Eyzaguirre², Gustavo Bravo Orellana² \\ ${ }^{1}$ Químico Farmacéutica; ${ }^{2}$ Facultad de Farmacia y Bioquímica-UNMSM
}

\section{RESUMEN}

Se revisaron 2843 pesquisas de productos farmacéuticos y afines, realizadas por el Equipo de Control y Vigilancia de Establecimientos de la DIGEMID de 2002 a 2006. Los análisis fisicoquímicos y microbiológicos fueron realizados por el Instituto Nacional de Salud y luego evaluados por el Equipo de Control y Vigilancia de Productos. Del total de productos pesquisados, se encontró que el $65 \%$ son conformes y $35 \%$ no conformes. Los resultados de los no conformes se clasificaron para determinar las principales observaciones a la calidad: Rotulado No Autorizado 40\%, Producto Deficiente $27 \%$, Crítico 17\%, Cambio de especificación 14\%, Sin Registro Sanitario 1\% y Forma de Presentación No Autorizada 1\%. De las pesquisas realizadas: $45 \%$ fueron medicamentos de marca, 30\% medicamentos genéricos, $10 \%$ material médico y $9 \%$ cosméticos. Resultaron conformes: Medicamentos de marca $69 \%$, medicamentos genéricos $64 \%$, material médico $60 \%$ y cosméticos $68 \%$. Respecto al tipo de Establecimiento Farmacéutico que presentan conformidad en sus productos tenemos: Laboratorios $69 \%$, Droguerías 62\% e Importadoras 67\%. Según país de procedencia, los conformes fueron $71 \%$ nacionales y $59 \%$ extranjeros.

Palabras clave: DIGEMID, Reglamento, Pesquisa, Calidad de medicamento, Vigilancia sanitaria.

\section{SUMMARY}

2843 inquiries were checked, pharmaceuticals and related products, made by the Control and Monitoring Equipment Establishments of DIGEMID from 2002 to 2006. The physicochemical and microbiological analyses were realized by the National Institute of Health and then evaluated by the Control and Monitoring Product. Of the whole of products were found that $65 \%$ are satisfied and $35 \%$ did not comply. The results of the non-compliant were classified to determine the main observations about quality: Unauthorized labeling 40\%, Deficient product $27 \%$, Critical $17 \%$, Specification change $14 \%$, Without sanitary record $1 \%$ and Unauthorized presentation form $1 \%$. In their investigation: $45 \%$ were brand name drugs, generic drugs 30\%, 10\% medical supplies and 9\% cosmetics. Were in conformity: brand drugs $69 \%$, generic drugs $64 \%$, medical supplies $60 \%$ and cosmetics $68 \%$. Regarding the type of pharmaceutical establishment in accordance presenting their products are: labs $69 \%$, drugstores $62 \%$, importing $67 \%$. By country of origin, the line were $71 \%$ home and $59 \%$ foreign.

Key words: DIGEMID, Regulation, Inquiry, Medicine quality, Sanity alertness.

\section{INTRODUCCIÓN}

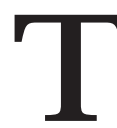

odo producto farmacéutico recorre un largo camino desde su elaboración hasta su utilización por el paciente; siendo posible que en algún lugar de este camino pueda verse afectada la calidad, por factores intrínsicos o extrínsecos. Debido a la seriedad de este problema, la Organización Mundial de la Salud (OMS) y la Organización Panamericana de la Salud (OPS) han alertado a las autoridades sanitarias sobre la existencia de productos alterados y la necesidad de retirarlos del mercado. Así, se han reportado casos de detección de partículas negras en un lote de gentamicina en Alemania en 1997; en 1993, en el Reino Unido, se retiraron del mercado cuatro lotes de una formulación de mitoxantrona inyectable porque se puso en duda la esterilidad del producto ${ }^{(1-4)}$.

Debido a estas situaciones, los organismos reguladores de cada país han definido procedimientos legales para retirar del mercado los productos defectuosos, estableciendo programas de control para detectar observaciones a la calidad de los medicamentos ${ }^{(1)}$, verificar que se cumplan las especificaciones de calidad establecidas en farmacopeas internacionales reconocidas y asegurar que los medicamentos lleguen a los pacientes en condiciones de calidad, seguros y eficaces ${ }^{(5,6)}$.

En el Perú, las reglamentaciones existentes: La Ley General de Salud № 26842 y el Reglamento para el Registro, Control y Vigilancia Sanitaria de Productos Farmacéuticos y Afines - Decreto Supremo № o10-97SA, con sus respectivas modificaciones y ampliatorias establecen que, el Registro Sanitario de productos far- 
macéuticos y afines se otorga sólo por referencia, es decir, si se encuentra registrado en cualquier país del mundo, y por lo tanto cuenta con un Certificado de Libre Venta. Este sistema no permite realizar ningún tipo de evaluación sobre la eficacia, seguridad y calidad de los productos, permitiendo la existencia en el mercado de medicamentos de valor terapéutico cuestionado ${ }^{(7-9)}$.

En el presente trabajo se evaluó la calidad de los productos farmacéuticos y afines del mercado peruano para evitar problemas como el ocurrido en los años 1980 a 1985, donde murieron niños a quienes se administraron las llamadas "bolsitas salvadoras" (sales de rehidratación oral) importadas de Estados Unidos de Norteamérica; en la investigación se descubrió que habían sido fabricadas en un estacionamiento sin ninguna exigencia sanitaria. Otro ejemplo es la adquisición de sulfato de bario argentino de calidad industrial (no medicinal) para radiodiagnóstico por un hospital de la Seguridad Social ${ }^{(10)}$.

\section{MATERIALES Y MÉTODOS}

\section{Materiales}

- Libros de pesquisas de los productos farmacéuticos y afines de los años 2002 al 2006, de la Dirección General de Medicamentos Insumos y Drogas (DIGEMID).

- Base de datos Perudis de la DIGEMID.

- Listado de Establecimientos Farmacéuticos contenida en la base de datos SISFAR de la DIGEMID.

- Ficha de Recolección de Datos.

\section{Método}

El presente es un estudio retrospectivo, transversal y descriptivo, cuyo objetivo fue determinar las principales observaciones a la calidad que presentan los productos farmacéuticos (medicamentos de marca, genéricos, y productos de origen biológico) y afines (productos dietéticos y edulcorantes, reactivos de diagnóstico, productos galénicos, productos naturales, cosméticos, productos sanitarios e instrumental y equipo de uso médico quirúrgico u odontológico), entre los meses de Octubre a Diciembre del 2007 en la Dirección de Control y Vigilancia Sanitaria (Área de Evaluación de Productos Farmacéuticos) de la DIGEMID.

\section{Muestra}

Se seleccionaron 2843 productos de los libros de pesquisas de productos farmacéuticos y afines de los años $2002-2006$.

\section{Criterios de Selección}

- Criterios de Inclusión: Pesquisas de los productos farmacéuticos y afines durante los años 2002 -2006 .

- Criterios de Exclusión: Productos que presentaban datos incompletos o cuyos resultados no se han remitido, por lo cual no están inscritos en el libro de pesquisas.

\section{Procedimiento}

- Se solicitaron las pesquisas realizadas por la DIGEMID entre 2002 a 2006.

- La toma de muestra se efectuó por duplicado, una se quedaba sellada por los inspectores en la empresa como contramuestra y la otra era remitida a DIGEMID. Luego se llenaba el Acta de Pesquisa de Productos Farmacéuticos y Afines en el libro de pesquisas del año correspondiente. El producto pesquisado es remitido al Área de Envío de Muestra, la cual se comunica con el laboratorio o droguería titular del Registro Sanitario.

- Los estudios de los análisis fisicoquímicos y microbiológicos fueron realizados en el Instituto Nacional de Salud y los resultados evaluados por el Área de Evaluación de Productos Farmacéuticos de la DIGEMID. El tiempo del análisis es según el tipo de producto a analizar; así, si son estériles 25 días y no estériles 15 días.

- Se analizaron 2843 productos farmacéuticos y afines, registrándose en la Ficha de Recolección de Datos diseñada, la siguiente información: Nombre del producto, país, titular del registro sanitario, resultados y las columnas con los códigos respectivos.

\section{RESULTADOS}

Tabla 1. Cantidad de Productos Pesquisados por año.

\begin{tabular}{ccc}
\hline & Productos Pesquisados & \\
\hline Año & $\begin{array}{c}\text { Número de } \\
\text { productos }\end{array}$ & $\begin{array}{c}\text { Porcentaje } \\
\text { (\%) }\end{array}$ \\
\hline 2002 & 474 & 16,67 \\
2003 & 529 & 18,61 \\
2004 & 485 & 17,06 \\
2005 & 568 & 19,98 \\
2006 & 787 & 27,68 \\
Total & 2843 & 100,00 \\
\hline
\end{tabular}


Tabla 2. Resultados de la conformidad de los productos pesquisados por año.

\begin{tabular}{ccccccc}
\hline \multirow{2}{*}{ Resultado } & \multicolumn{7}{c}{ Años } & \multirow{2}{*}{ Total } \\
\cline { 2 - 6 } & $\mathbf{2 0 0 2}$ & $\mathbf{2 0 0 3}$ & $\mathbf{2 0 0 4}$ & $\mathbf{2 0 0 5}$ & $\mathbf{2 0 0 6}$ & \\
\hline Conforme & 347 & 296 & 269 & 384 & 559 & 1855 \\
No conforme & 127 & 233 & 216 & 184 & 228 & 988 \\
Total & 474 & 529 & 485 & 568 & 787 & 2843 \\
\hline
\end{tabular}

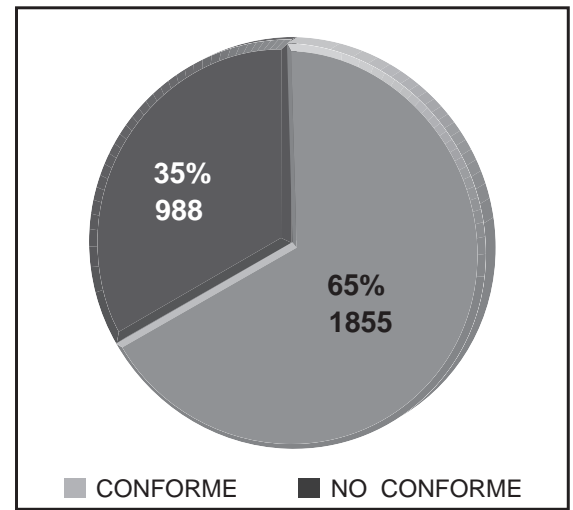

Tabla 3. Número de muestras pesquisadas según tipo de producto.

Figura 1. Resultados de las Pesquisas

\begin{tabular}{|c|c|c|c|c|c|c|c|}
\hline \multirow{2}{*}{ Rubro } & \multicolumn{5}{|c|}{ Año } & \multirow{2}{*}{ Total } & \multirow{2}{*}{$\begin{array}{c}\text { Porcentaje } \\
\text { (\%) }\end{array}$} \\
\hline & 2002 & 2003 & 2004 & 2005 & 2006 & & \\
\hline Medicamentos de Marca & 203 & 195 & 197 & 280 & 386 & 1261 & 45 \\
\hline Medicamento Genérico & 153 & 169 & 172 & 139 & 214 & 847 & 30 \\
\hline Producto Natural & 2 & 16 & 5 & 19 & 20 & 62 & 2 \\
\hline Material médico & 44 & 63 & 56 & 60 & 66 & 289 & 10 \\
\hline Cosmético & 51 & 70 & 29 & 39 & 67 & 256 & 9 \\
\hline Galénico & 9 & 3 & 10 & 7 & 6 & 35 & 1 \\
\hline Dietético & 6 & 10 & 7 & 13 & 16 & 52 & 2 \\
\hline Producto Biológico & 0 & 2 & 6 & 1 & 1 & 10 & 0 \\
\hline Producto Sanitario & 6 & 1 & 2 & 5 & 10 & 24 & 1 \\
\hline Reactivo de Diagnóstico & 0 & 0 & 1 & 5 & 1 & 7 & 0 \\
\hline Total & 474 & 529 & 485 & 568 & 787 & 2843 & 100 \\
\hline
\end{tabular}

Tabla 4. Número de productos evaluados por tipo de establecimiento.

\begin{tabular}{cccccc}
\hline Año & Laboratorio & Droguería & Importadora & Otros & Total \\
\hline 2002 & 178 & 278 & 17 & 1 & 474 \\
2003 & 217 & 294 & 17 & 1 & 529 \\
2004 & 205 & 267 & 11 & 2 & 485 \\
2005 & 235 & 324 & 9 & 0 & 568 \\
2006 & 277 & 489 & 21 & 0 & 787 \\
Total & 1112 & 1652 & 75 & 4 & 2843 \\
\hline
\end{tabular}

\section{DISCUSIÓN}

En el Perú contamos con tres documentos importantes: La Ley General de Salud № 26842, publicada el 20 de julio de 1997; el Reglamento para el Registro, Control y Vigilancia Sanitaria de Productos Farmacéuticos y Afines del 24 de diciembre de 1997 y la Directiva de Pesquisas de Productos Farmacéuticos y Afines del 11 de noviembre de $1998^{(8,11,12)}$.

Los artículos № 50 y 92 de la Ley General de
Salud, Capítulo III: De los productos farmacéuticos y galénicos, y de los recursos terapéuticos naturales y Capítulo V: De los alimentos, bebidas, productos cosméticos y similares, insumos, instrumental y equipo de uso médico-quirúrgico u odontológico, productos sanitarios y productos de higiene personal y doméstica, antes de la modificatoria de 2009, señalaba los requisitos para la obtención del Registro Sanitario. Esto ha sido tomado para la elaboración de los reglamentos antes citados, donde se establecía que en base a una 
simple documentación y en tan sólo siete días se puede inscribir o reinscribir un producto farmacéutico o afín y, es más, si pasan los siete días y no hay respuesta, esto se toma como un silencio administrativo positivo.

En la DIGEMID, los Registros Sanitarios de Productos Farmacéuticos otorgados del 2002 a 2006 disponen de una base de datos digital (Perudis), mientras que los afines se registraron manualmente del 2002 al 2004; según estos el total de productos farmacéuticos y afines registrados el 2006 es de 8112, tanto registros nuevos como reinscripciones. Este alto número de Registros Sanitarios al año contribuye al uso irracional de los medicamentos, sumado a la escasa presencia del Químico Farmacéutico en boticas y farmacias, quien es la persona más adecuada en la orientación del uso.

Sobre Productos Pesquisados, es evidente el incremento en el número de pesquisas realizadas en el 2002 (474 pesquisas) respecto al 2006 ( 787 pesquisas) (Tabla 1), considerando que la Ley General de Salud establece que el porcentaje de pesquisas debe ser el 10\% del total de productos farmacéuticos y afines registrados por año. Las pesquisas que se realizan están en relación con la Política General de Medicamentos establecida a nivel del Ministerio de Salud.

Respecto a la evaluación efectuada a los productos pesquisados, se observa un incremento en los resultados conformes a través de los años; lo cual se explica debido al control realizado mediante las inspecciones y auditorias de certificación, verificando el cumplimiento de las Buenas Prácticas de Manufactura (BPM) en las empresas manufacureras nacionales, así como el cumplimiento de las Buenas Prácticas de Almacenamiento (BPA) para el resto de las empresas (Tabla 2). En relación a los productos con resultados no conformes, el mayor número de problemas son por Rotulado, mientras por el contrario, la Forma de presentación no autorizada ha disminuido a tres desde el 2002 y cero en 2006. Los resultados de las pesquizas y de No conformes se muestran en las figuras 1 y 2.

En cuanto a los Productos críticos, se observa una fluctuación de los resultados del 2002 al 2006; encontrándose observaciones en ensayos microbiológicos, en la identificación, disolución y concentración del principio activo. La mayoría de estos productos corresponden a medicamentos de marca y genéricos (AINES, antibióticos penicilínicos y cefalosporínicos).

En relación a los Resultados deficientes, se observa que ha habido una disminución desde 15,88 \% en el 2003 a 3,43 \% el 2006. Los tipos de productos con mayores observaciones en la calidad, son los medicamentos de marca, genéricos y equipo e instrumental de uso médico quirúrgico y odontológico entre los años 2003 a 2005, los cosméticos en $2003 \mathrm{y}$ los productos naturales en 2006 (Tabla 3).

Los resultados por observaciones en el rotulado nos muestran que ha habido un ligero descenso desde el 2003, con $17,2 \%$, al 2006 con 13,72\%; porque se han realizado cambios oportunos, comunicándolos a la DIGEMID (Tabla 5).

La disgregación de los resultados no conformes entre 2002 a 2006, muestra en primer lugar un $40 \%$ en Rotulado no autorizado; además, es preocupante que las Observaciones críticas presenten un $17 \%$ y Por deficiencia un $27 \%$. Esto podría traer problemas de salud pública por tratarse de observaciones graves que afectan la seguridad y eficacia del producto (Tabla 5).

En relación a la clase de productos que fueron pesquisados del 2002 a 2006, corresponde a: medicamentos de marca $45 \%$, medicamentos genéricos 30\%, material médico quirúrgico u odontológico $10 \%$ y cosmético $9 \%$, y las observaciones a la calidad corresponden a: medicamentos de marca $31 \%$, medicamentos genéricos $36 \%$, material médico u odontológico $40 \%$ y cosméticos 32\% (Tabla 3).

Por el tipo de establecimiento farmacéutico donde se pesquisó, se encontró: droguerías $58 \%$, laboratorios $39 \%$ e importadoras $3 \%$; de éstos, el porcentaje con observaciones al tipo de calidad: críticas, deficiente, cambio de especificación y por rotulado, corresponden a droguerías $38 \%$, laboratorios 31\% e importadoras $33 \%$. Para el caso de los laboratorios, hubo un aumento del número de conformes en relación a los no conformes; estos últimos han disminuido con respecto a los 


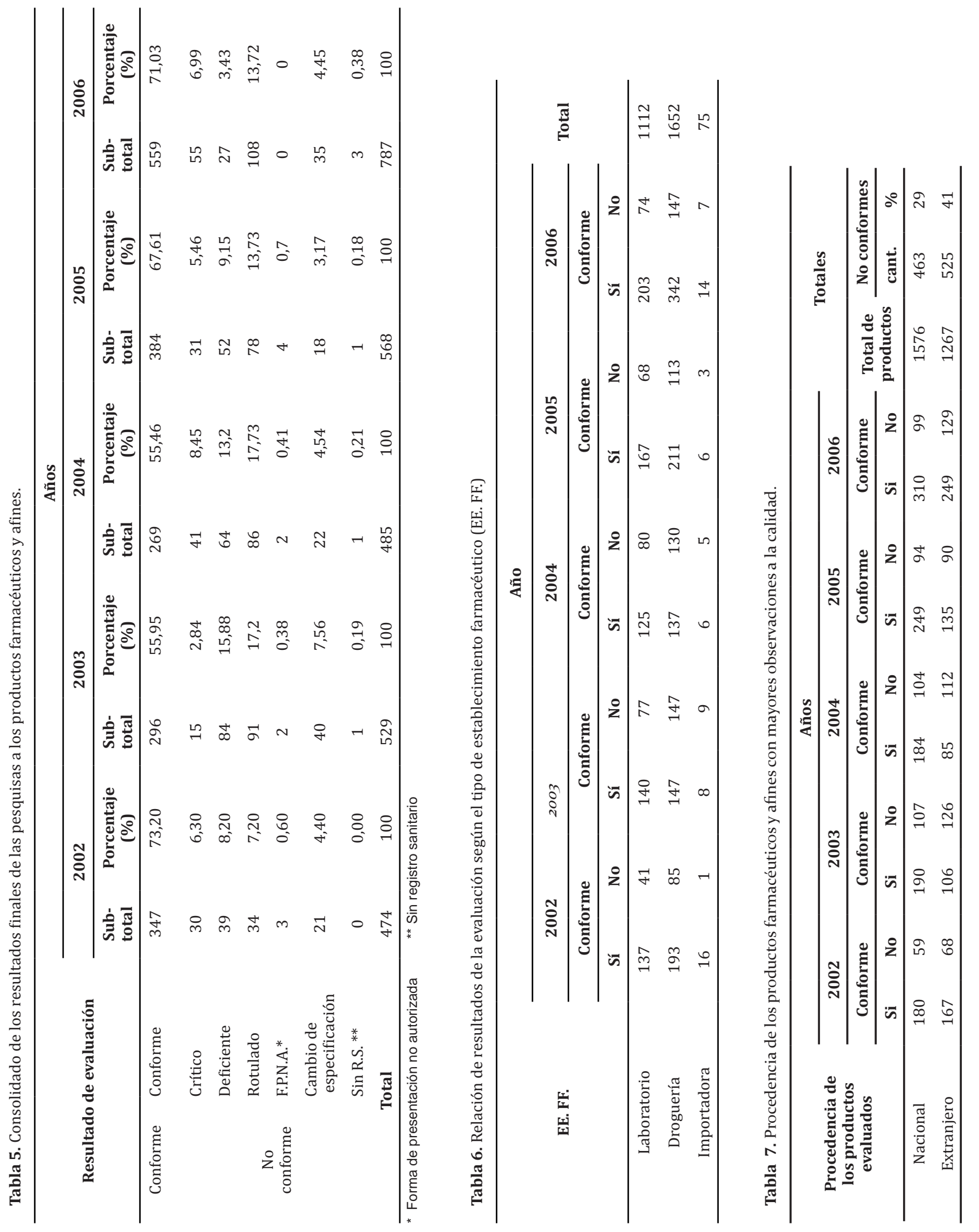


años anteriores debido al mayor control que se realiza mediante las inspecciones de seguimiento; caso contrario ocurre con las importadoras y droguerías donde no se exige la presencia permanente del Químico Farmacéutico, quien debe verificar las condiciones de almacenamiento de los productos a fin de asegurar la calidad de los mismos (Tabla 6).

Según la procedencia, el $55 \%$ de los productos son de origen nacional y $45 \%$ son extranjeros. En relación a los primeros, del 2002 al 2006 ha habido un incremento de los resultados conformes; los resultados de no conformes se han mantenido constantes del 2003 al 2006. Para los de origen extranjero, en los resultados conformes se observa un descenso entre los años 2003 y 2004; incrementándose del 2005 al 2006. El porcentaje de los no conformes, del 2002 al 2006, para los de origen nacional fue de $29 \%$ y de $41 \%$ para los extranjeros (Tabla 7 ).

En el Perú, hasta la realización del presente estudio, la Ley General № 26842, artículo 50 y 92, establecía que "La inscripción en el Registro Sanitario de productos farmacéuticos y afines es automática, con la sola presentación de los documentos establecidos en dicho dispositivo, teniendo la autoridad de salud un plazo máximo de 7 días útiles para expedir el documento que acredite el número de registro"; situación que ha sido modificada evitando que nuestro país sea observado al tener la legislación más permisiva del mundo, con un proceso de registro poco confiable.

\section{CONCLUSIONES}

En la evaluación de la calidad de los productos farmacéuticos y afines pesquisados se encontró: Rotulado noautorizado $40 \%$, Productodeficiente $27 \%$, Crítico $17 \%$, Cambio de especificación 14\%, Sin Registro Sanitario 1\% y Forma de presentación no autorizada 1\%. De estos, 31\% son medicamento de marca, $36 \%$ son genéricos, $40 \%$ material médico; mientras que el 32\% de los cosméticos presentan resultados de no conformidad.

Los productos con observaciones a la calidad, por tipo de establecimiento farmacéutico fueron: laboratorios 31\%, droguerías 38\% e importadoras 33\%.

De otro lado, de los productos farmacéuticos y afines pesquisados no conformes, $29 \%$ son de procedencia nacional y $41 \%$ extranjera; siendo la India el país con más observaciones.

\section{REFERENCIAS BIBLIOGRÁFICAS}

1. Grupo de Trabajo de la Revista Panamericana de Salud Pública. Programa de detección de fallas de calidad en los medicamentos comercializados. OPS (Washington)
1999 septiembre; 6 (3): 20-22.

2. Barros J. Las políticas farmacéuticas. Ediciones UNESCO, Ciudad de México 2002 marzo; 4 (1): 5-7.

3. Agencia Europea de Medicamentos. Grupo de trabajo sobre control e inspección de los medicamentos de la Comisión Europea, Guía comunitaria de normas de correcta fabricación de la EMEA; 2001. Serie de Guía: 180.

4. Organización Mundial de la Salud. La OMS intensifica su lucha contra los medicamentos falsificados. 2003 Noviembre. Disponible en: http://www.who.int/mediacentre/news/releases/2003/pr85/es/index (Fecha de acceso 23 de junio de 2007).

5. Ministerio de Salud. Medicamentos y calidad. 2004. Disponibleen:http://www.msal.gov.ar/htm/site/Genericos/ site2/calidad.asp (Fecha de acceso 23 de junio de 2007).

6. Organización Mundial de la Salud. Seguridad de los Medicamentos. 2005. Disponible en: http://www.who. int/mediacentre/factsheets/fs293/es/index.htm (Fecha de acceso 23 de junio de 2007).

7. Decreto Supremo $\mathrm{N}^{\circ}$ 023-2005-SA del 29 de diciembre: Reglamento de Organización y Funciones del Ministerio de Salud (Normatividad Institucional de la DIGEMID, número 33 del o1 de enero de 2003).

8. Resolución Ministerial $\mathrm{N}^{\circ}$ 437-98-SA/DM del o6 de noviembre: Directiva de Pesquisas de Productos Farmacéuticos y Afines (Normatividad Institucional de la DIGEMID, número 92 del 11 de noviembre de 1998)

9. Carvajal J, Flores I. Ensayos de calidad sobre medicamentos existentes en el mercado nacional: Muestreo en el primer trimestre 1992. Tesis para optar al Titulo Profesional de Químico Farmacéutico. Facultad de Farmacia y Bioquímica, Universidad nacional Mayor de San Marcos. Lima, 1992.

10. Orihuela C. Calidad de los Medicamentos. Foro Salud 2002. disponible en: http://wari.rcp.net.pe/FRE/forosalud/FINAL/pdfs/Rel_Med.pdf (Fecha de acceso 23 de junio de 2007).

11. Ley $\mathrm{N}^{\circ} \mathbf{2 6 8 4 2 - 0 2}$ del 27 de enero: Ley General de Salud

12. Decreto Supremo $N^{\circ}$ o10-97-SA y Modificatoria DS $N^{\circ}$ oo4-oo-AS, oo6-o1-AS y o05-04-SA: Reglamento para el Registro, Control y Vigilancia Sanitaria de Productos Farmacéuticos y Afines.

Manuscrito recibido el: 13/11/2009

Aceptado para su publicación el: 07/01/2010

\section{Correspondencia:}

Dr. José Juárez Eyzaguirre

Jr. Los Tulipanes 343 - Lima 12

E-mail: jjuareze@unmsm.edu.pe 